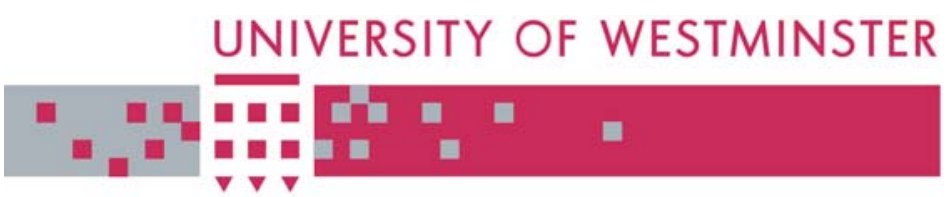

\title{
WestminsterResearch
}

http://www.wmin.ac.uk/westminsterresearch

\section{A blind implementation of multi-dimensional matched filtering in a Maximum-Likelihood receiver for SIMO channels.}

\section{Adem Coskun}

Izzet Kale

School of Electronics and Computer Science

Copyright (C) [2009] IEEE. Reprinted from the proceedings of the IEEE Intrumentation and Measurement Technology Conference (I2MTC '09). IEEE, pp. 633-637. ISBN 9781424433520.

This material is posted here with permission of the IEEE. Such permission of the IEEE does not in any way imply IEEE endorsement of any of the University of Westminster's products or services. Personal use of this material is permitted. However, permission to reprint/republish this material for advertising or promotional purposes or for creating new collective works for resale or redistribution to servers or lists, or to reuse any copyrighted component of this work in other works must be obtained from the IEEE. By choosing to view this document, you agree to all provisions of the copyright laws protecting it.

The WestminsterResearch online digital archive at the University of Westminster aims to make the research output of the University available to a wider audience. Copyright and Moral Rights remain with the authors and/or copyright owners.

Users are permitted to download and/or print one copy for non-commercial private study or research. Further distribution and any use of material from within this archive for profit-making enterprises or for commercial gain is strictly forbidden.

Whilst further distribution of specific materials from within this archive is forbidden, you may freely distribute the URL of the University of Westminster Eprints (http://www.wmin.ac.uk/westminsterresearch).

In case of abuse or copyright appearing without permission e-mail wattsn@wmin.ac.uk. 


\title{
A Blind Implementation of Multi-Dimensional Matched Filtering in a Maximum-Likelihood Receiver for SIMO Channels
}

\author{
Adem Coskun and Izzet Kale \\ Applied DSP and VLSI Research Group \\ University of Westminster \\ London, W1W 6UW, United Kingdom \\ adem@alptron.com,kalei@westminster.ac.uk
}

\begin{abstract}
In order to establish the optimal receiver strategy, in terms of error rate for Single Input Multi Output (SIMO) wireless channels, the Maximum Likelihood (ML) detection should be performed following a multi-dimensional matched filter. However, the implementation of the matched filter and the ML detection both need the estimation of the channel impulse response in advance. In this work, we propose a novel method to establish the matched filters of the SIMO channel blindly alongside a three-step technique for the blind and adaptive ML detection of the symbol vector. With the use of the novel method, the system will benefit from the bandwidth efficiency point of view due to the use of blind schemes. The Constant Modulus Algorithm is utilized to perform the blind matched filtering operation and later Least Mean Squared algorithm is introduced for further correction on the matched filter estimate. The blindly estimated matched filters are incorporated into the ML detector so that the transmitted symbols are found and therefore the channel is equalized. Simulations are provided to present the equalization performance and convergence speed of the novel technique.
\end{abstract}

\section{INTRODUCTION}

The use of spatial diversity, to improve the error rate performance, is one of the promising solutions to the reliability problems of wireless communication systems. Placing sufficiently spaced antennas at the receiver end would generate several independent channels and therefore enable reception of replicas of the transmitted signal. The system comprising a single transmit antenna with multiple antennas employed at the receiver is called SIMO, where the additional receiving antennas benefit the system with additional spatial diversity.

Many of the techniques used to establish the channel coefficients of a SIMO channel depend on the transmission of training signals in order to inform the receiver of the channel. This approach is inherently inefficient and wastes the bandwidth of the communication channel as well as resources at both ends. That is why a blind scheme, adapted for such wireless communication scenarios, would definitely improve the system efficiency.

The blind methods proposed for SIMO channel models involve matrix decompositions, which are computationally expensive, such as Singular Value Decomposition (SVD) and Eigen Value Decomposition (EVD) (for further reading on decomposition type channel estimation please refer to
[1], [2]). In the work of Tugnait et. al. [3], higher order cumulant functions of the received data are used to estimate the Channel Impulse Response (CIR) which is then used to construct a linear equalizer. However increasing the order of cumulant functions will eventually lead to the need for long data records for accurate estimation of Higher Order Statistics (HOS). Ozcelik et. al [4] proposed a novel method for blind Maximum Likelihood Sequence Equalization (MLSE) of Single Input Single Output (SISO) channels, using the matched filter estimates obtained with a computationally simple algorithm, that is the Constant Modulus Algorithm (CMA) [5], without any need for matrix decomposition operation in addition to processing only with the Second Order Statistics (SOS) of the received data. Therefore the main objective of our work was set to adapt the receiver in [4] for SIMO channels for better diversity gain and to benefit from the method's computational convenience while obtaining the matched filter estimates of each channel.

It is also well known that the optimal receiver strategy in terms of error rate for SIMO channels is to perform ML detection following Multi-dimensional Matched Filtering [3]. Multi-dimensional matched filtering operation corresponds to performing matched filtering at each single antenna and then combining their outputs to obtain a single stream. Therefore we can claim that; If the matched filter estimates can correctly be obtained, then they can further be incorporated into a Maximum Likelihood Sequence Detection (MLSD) setup with the Viterbi Algorithm (VA) to estimate the transmitted information sequence for optimum performance.

In Section II we will first introduce the system model assumed for the work reported in the rest of the paper and how the methodology in [1] was adapted for SIMO, followed by how the DFE and MLSD types of equalizations are performed blindly. A three step technique will later be explained where the channel estimates are further corrected by the use of Least Mean Squared (LMS) algorithm. The last section is dedicated to the simulation results.

\section{SySTEM MODEL AND NOVEL BLIND MLSD SETUP}

\section{A. System Model}

We have assumed a single transmitter/receiver scenario, where $\mathrm{M}_{\mathrm{r}}$ number of antennas are placed at the receiver with 
the transmitter having a single antenna (i.e $\mathrm{M}_{\mathrm{t}}=1$ ). Therefore in total there are $\mathrm{M}_{\mathrm{r}}$ channels, through which the signal transmission is conducted. The channels in between each receiving antenna and the transmitter are frequency selective. Each of the $\mathrm{M}_{\mathrm{r}}$ frequency selective channels is assumed to be composed of $\mathrm{L}$ discrete coefficients. The SIMO channel for any one of the $\mathrm{M}_{\mathrm{r}}$ receive antennas can be written as;

$$
\mathbf{y}_{\mathrm{i}}[k]=\mathbf{H}_{\mathrm{i}} \mathbf{s}[k]+\mathbf{n}_{\mathrm{i}}[k] \quad \text { for } \mathrm{i}=1, \cdots, \mathrm{M}_{\mathrm{r}}
$$

where $\mathbf{y}_{\mathrm{i}}$ is the stacked vector of received signals and $\mathbf{n}_{\mathrm{i}}$ is the stacked vector of noise components for the $i^{\text {th }}$ receive antenna. Both vectors $\mathbf{y}_{i}$ and $\mathbf{n}_{i}$ are of length Tx1 (corresponds to $\mathrm{T}$ times one), where $\mathrm{T}$ represents the number of taps of the equalizer placed following a receive antenna. Noise is assumed to be Additive White Gaussian (AWGN), which has independent and identically distributed (i.i.d.) samples. $\mathbf{s}$ is the vector of complex data symbols. The channel matrix $\mathbf{H}_{\mathrm{i}}$ is a Toeplitz matrix in the form;

$$
\mathbf{H}_{\mathrm{i}}=\left[\begin{array}{cccccc}
h_{\mathrm{i}}[\mathrm{L}-1] & \cdots & h_{\mathrm{i}}[0] & 0 & \cdots & 0 \\
\vdots & \ddots & \ddots & \ddots & \ddots & \vdots \\
0 & \cdots & 0 & h_{\mathrm{i}}[\mathrm{L}-1] & \cdots & h_{\mathrm{i}}[0]
\end{array}\right]
$$

Each row of $\mathrm{H}_{\mathrm{i}}$ contains the multipath components of the $\mathrm{i}^{\text {th }}$ channel that is $\mathbf{h}_{\mathrm{i}}=\left\{h_{\mathrm{i}}[\mathrm{L}-1] \quad \cdots \quad h_{\mathrm{i}}[0]\right\}[6$, page 139].

\section{B. Blind Matched Filter Estimation}

Fig. 1 presents the implementation of a linear DFE for a SIMO communication system, where the channel of each receive antenna is matched filtered. The DFE is called "linear" due to the fact that the Decision Device (DD) is placed after the feedback filtering operation. Each matched filter, $\mathbf{m}_{i}$, is the conjugated time-reversed version of its corresponding channel $\mathbf{h}_{i}$, i.e. $\mathbf{m}_{\mathrm{i}}[k]=\mathbf{h}_{\mathrm{i}}^{*}[\mathrm{~L}-k]$. As can be seen, every receive antenna needs a feedforward filter $\mathbf{f}_{i}$, while a single feedback filter $\mathbf{b}$ is enough to remove the post-ISI from the signal, generated by adding the outputs of the feedforward filters [7]. The block, which has the filter b at its feedback, is denoted by $\mathrm{B}$, is shown in Fig. 1. Deploying linearity, the sub-block B in Fig. 1 can be moved to be before the summation of the feedforward filters $\mathbf{f}_{\mathrm{i}}$ and the matched filter can be placed following B. Therefore Fig. 1 can be re-drawn as shown in Fig. 2 .

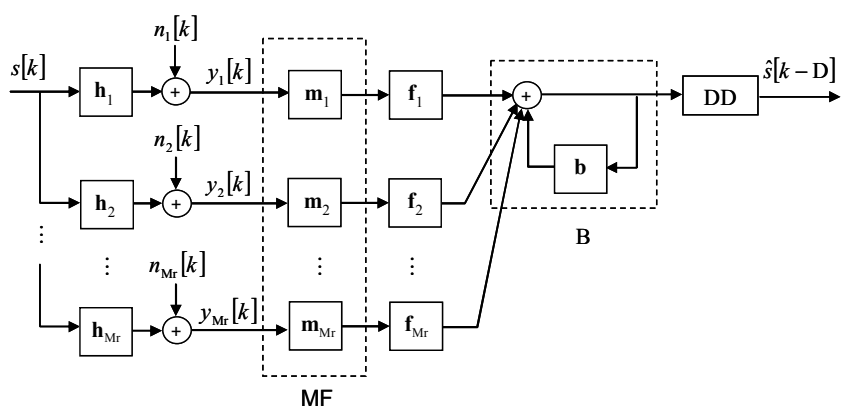

Fig. 1. Linear SIMO-DFE Receiver with matched filtering

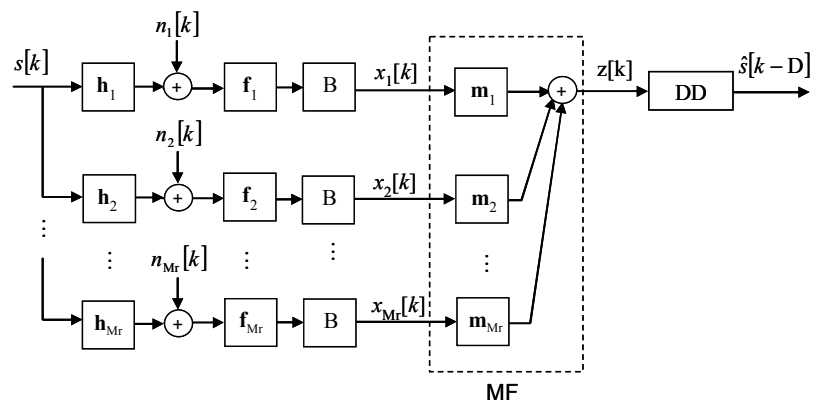

Fig. 2. SIMO-DFE Receiver after rearrangement of receiver blocks

The block with dashed lines in Fig. 2 (i.e. MF) is where the multi-dimensional matched filtering is performed. It can be viewed as a system of $\mathrm{M}_{\mathrm{r}}$ discrete Finite Impulse Response (FIR) filters, the output of which are added down to a single output after filtering. For our work, MF is assumed to be a single FIR filter of length $\mathrm{M}_{\mathrm{r}} \mathrm{xL}$, the coefficients of which are adaptively updated by the CMA algorithm.

Calling the MF vector $\mathbf{M}$, the CMA can be used for its update as formulated below [8]

$$
\mathbf{M}(n+1)=\mathbf{M}(n)+\mu \mathbf{x}[n] z[n]\left(|z[n]|^{2}-1\right)^{2}
$$

providing that a data symbol constellation having a constant squared-modulus of one (i.e. $|s[k]|^{2}=1$ ) is preferred. In equation (3) $n$ represents the iteration number. $\mathbf{M}(n)$ is the MF vector at the $n^{\text {th }}$ iteration and similarly $z[n]$ is the output of filter $\mathbf{M}$ at the $n^{\text {th }}$ iteration. $\mu$ is a small positive step size and $\mathbf{x}[n]$ is the concatenated vector of processed symbols by the DFE filters corresponding to the $n^{\text {th }}$ MF output;

$$
\begin{aligned}
\mathbf{x}[n]=\left[x_{1}[n], x_{1}[n-1], \cdots, x_{1}[n-L+1],\right. \\
\left.x_{2}[n], x_{2}[n-1], \cdots, x_{\mathrm{Mr}}[n-L+1]\right]
\end{aligned}
$$

It should also be noted that; If the iterations to update $\mathbf{M}$ are made periodically at every symbol period so that $n$ is incremented as $k$ does, then the two index variables $k$ and $n$ can be regarded as the same.

\section{Blind calculation of the SIMO-DFE and the SIMO- MLSD parameters}

The optimal feedforward and feedback filter weights of a SIMO-DFE can be calculated by simply multiplying the correlation matrices $\mathbf{R}_{\mathrm{a}}$ and $\mathbf{R}_{\mathrm{b}}$,

$$
\widetilde{\mathbf{g}}=\mathbf{R}_{\mathrm{a}} \mathbf{R}_{\mathrm{b}}^{-1}
$$

where, the $1 \mathrm{x}\left(\mathrm{M}_{\mathrm{r}} \mathrm{T}+\mathrm{F}\right)$ sized vector $\widetilde{\mathbf{g}}=[\mathbf{f} ;-\mathbf{b}]$ is composed of the concatenated feedforward filters (i.e. $\mathbf{f}=\left[\mathbf{f}_{1} \cdots \mathbf{f}_{\mathrm{Mr}}\right]$ ) of length $1 \mathrm{xM}_{\mathrm{r}} \mathrm{T}$ and the feedback filter of length $1 \mathrm{xF}$ [6, page 146]. The calculation of matrices $\mathrm{R}_{\mathrm{a}}$ and $\mathrm{R}_{\mathrm{b}}$ needs the channel matrix to be known. For our blind receiver, the channel, which is effective over the DFE filter, is the "channel+matched filter" combination as in Fig. 1. 
For that reason, the effective channel over branch i can be written as

$$
h_{\mathrm{i}}^{\text {eff }}[k]=\sum_{j=0}^{\mathrm{L}-1} h_{\mathrm{i}}[j] m_{\mathrm{i}}[k-j]=\sum_{j=0}^{\mathrm{L}-1} h_{\mathrm{i}}[j] h_{\mathrm{i}}^{*}[(\mathrm{~L}-1)-k+j]
$$

It can be realized from (6) that the effective channel corresponds to the autocorrelation of the vector $\mathbf{h}_{\mathrm{i}}$. In practice the autocorrelation can be blindly estimated using sample averages, written as

$$
\begin{gathered}
\hat{h}_{\mathrm{i}}^{\text {eff }}[k]=\sum_{j=0}^{\mathrm{N}} y_{\mathrm{i}}[j] y_{\mathrm{i}}^{*}[j+k] /\left(\mathrm{K} \sigma_{s}^{2}\right) \text { for } k \neq 0 \\
\hat{h}_{\mathrm{i}}^{\text {eff }}[0]=\left(\sum_{j=0}^{\mathrm{N}} y_{\mathrm{i}}[j] y_{\mathrm{i}}{ }^{*}[j] /\left(\mathrm{K} \sigma_{s}{ }^{2}\right)\right)-\sigma_{n}{ }^{2} \text { for } k=0
\end{gathered}
$$

which enables the calculation of the autocorrelation of the channel by manipulating the channel output without any need for a priori channel information, where $\mathrm{K}$ is the number of data symbols to be used for the estimation of the autocorrelation vector. $\mathrm{K}$ can be a few thousand for better estimation. In equations (7) and (8), $\sigma_{n}{ }^{2}$ and $\sigma_{s}{ }^{2}$ are the noise variance and the symbol variances respectively. Because Additive White Gaussian Noise (AWGN) is assumed to be independent and identically distributed, its variance only appears as a term added to the $0^{\text {th }}$ autocorrelation estimate as can be clearly seen from equation (8) where $k$ is set to 0 in (7).

In [4], it has been shown that the weights of an adaptive filter, using the CMA, can effectively converge to the matched filter equivalent of the channel, if the matched filter itself is the sole unknown in the receiver. Therefore, once the DFE filter weights are obtained by (5) after finding the unknown effective channel, it is guaranteed that the adaptive filter $\mathbf{M}$ will converge to the matched filters of unknown channels by iterating over equation (3).

The blind receiver structure constructed so far is called the blind SIMO-DFE. For optimal performance in the error rate sense, the receiver in Fig. 2 is adapted as in Fig. 3. In order to do so, the feedforward and feedback filters are disconnected from the receiver and the DD is replaced by

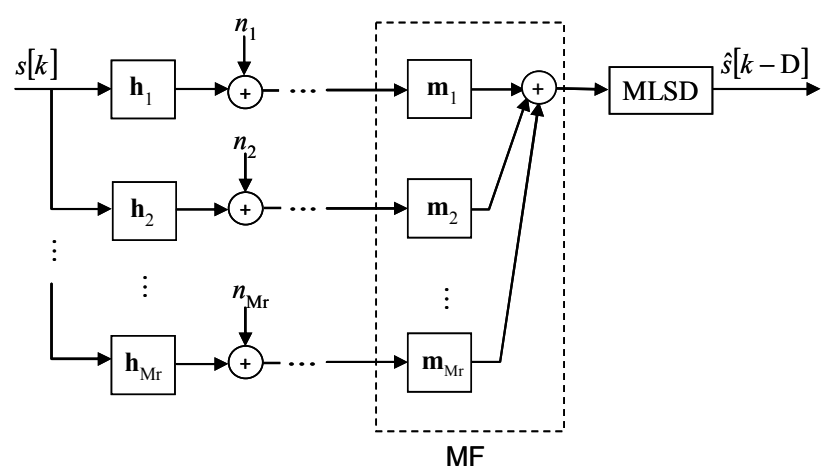

Fig. 3. Blind SIMO-MLSD setup after the removal of feedforward and feedback filters the MLSD block, implementing the Viterbi Algorithm (VA) (For further reading on VA, refer to [9]). In this way the Multi-dimensional matched filter operation is utilized before the ML estimation. Therefore if it is intended to get optimum BER performance, it is only a simple switching operation to activate the MLSD setup providing that the VA is already available in the system.

Similarly to the DFE, the effective channel over the MLSD is the "channel+matched filter" combination as well. Due to combining the effective channels of the multiple antennas before the VA, the effective channel to be incorporated into the MLSD should be composed of the sum of all paths' effective responses (see (6) which leads to (9)).

$$
q^{e f f}[k]=\sum_{\mathrm{i}=1}^{\mathbf{M}_{\mathrm{r}}} \sum_{j=0}^{\mathrm{L}-1} h_{\mathrm{i}}[j] h_{\mathrm{i}}^{*}[(\mathrm{~L}-1)-k+j]=\sum_{\mathrm{i}=1}^{\mathbf{M}_{\mathrm{r}}} h_{\mathrm{i}}^{\text {eff }}[k]
$$

In Equation (9), $q^{\text {eff }}[k]$ represents the channel coefficients to be known for applying the VA. Note that the unknown effective channels are already found in equation (6) during the parameter calculations of the DFE. Therefore no further statistical manipulation is needed at the MLSD setup stage for the calculation of $q^{e f f}[k]$.

We now introduce a three-step technique for the blind and adaptive ML detection of the symbol vector, which is distorted due to the frequency selective nature of the wireless channel, by making use of multiple antennas. The technique consists the matched filter estimation, MLSD implementation by the VA and further corrections for better ML estimates;

1) The matched filter estimation is performed blindly by the SIMO-DFE receiver as given in Fig. 2 utilizing the CMA.

2) By disconnecting the feedforward and feedback filters as in Fig. 3, the ML estimates of the transmitted sequence is obtained by the VA.

3) The matched filter estimates are adaptively updated by the LMS algorithm over the decisions produced in the SIMO-MLSD setup.

The last step of the technique requires the use of the LMS algorithm, which can be formulated as follows [9],

$$
\begin{gathered}
\hat{\mathbf{h}}_{\mathrm{i}}(n+1)=\hat{\mathbf{h}}_{\mathrm{i}}(n)-\eta e_{\mathrm{i}}(n) \hat{\mathbf{s}}[n] \\
e_{\mathrm{i}}(n)=y_{\mathrm{i}}[n]-\hat{\mathbf{h}}_{\mathrm{i}}(n) \hat{\mathbf{s}}[n]
\end{gathered}
$$

where $\eta$ is the step size for the LMS algorithm and $e_{\mathrm{i}}(n)$ is the decision error made at the estimation of the $i^{\text {th }}$ channel. In order to obtain the decision error, the symbol vector $\mathbf{s}$, sent from the transmitter, has to be known by the receiver. Because there is no training sequence to be used for the LMS equation, at the third step of the technique, a vector of data symbol estimates ( $\hat{\mathbf{s}}$ ), already found from the VA, activated at the second step and are used as the training sequences [4].

To apply the LMS algorithm, an initial value of the channel impulse response should also be known which appears in equation (10) as $\hat{\mathbf{h}}_{\mathrm{i}}$. For the proposed system, $\hat{\mathbf{h}}_{\mathrm{i}}$ 
can be obtained by taking the conjugate of the time reversal of the matched filter estimates from the CMA method, already found in step 1 .

\section{SimULATION RESULTS}

Simulations have been conducted in the MATLAB v.7.4 environment to illustrate the Bit Error Rate (BER) performance of both proposed schemes (Blind SIMO-DFE and Blind SIMO-MLSD) and to show the convergence of the CMA coefficients to the actual matched filter coefficients.

For the simulations, we used four antennas at the receiver, with the same four channels given in two previous studies on blind estimation [1, Table II] and [10, Table I], and are reported as on Table I.

Unit energy QPSK symbol transmission is assumed from the single transmit antenna and the channels of each receive branch are scaled to unit norm prior to simulations. The noise added to the received signals is assumed to be white Gaussian i.i.d.

TABLE I. SET OF CHANNEL RESPONSES [1], [10]

\begin{tabular}{|c|c|c|c|c|}
\hline$k$ & $h_{1}[k]$ & $h_{2}[k]$ & $h_{3}[k]$ & $h_{4}[k]$ \\
\hline 0 & 0 & $-1.280-0.301 i$ & $1.617+2.385 i$ & $0.178+0.263 i$ \\
\hline 1 & $-1.023-0.501 i$ & $0.106+1.164 i$ & $1.477+1.850 i$ & $-0.482-0.523 i$ \\
\hline 2 & 0 & $-0.282+0.562 i$ & $0.371-1.001 i$ & $0.041-0.110 i$ \\
\hline 3 & $-0.227+0.487 i$ & $0.031-0.211 i$ & $0.336-0.866 i$ & $-0.110+0.271 i$ \\
\hline
\end{tabular}

For the simulation, parameters are chosen as; $\mathrm{T}=8$ (The order of each feed-forward filter of the DFE), $\mathrm{F}=2$ (The order of feed-back filter of DFE), $\mathrm{D}=4$ (Delay), $\mu=5 \times 10^{-3}$ (Step size for CMA).

The plots on Fig. 4 reveal that the novel blind receiver performs equalization close to the matched filter bound. A noteworthy result is obtained from the novel blind SIMO-

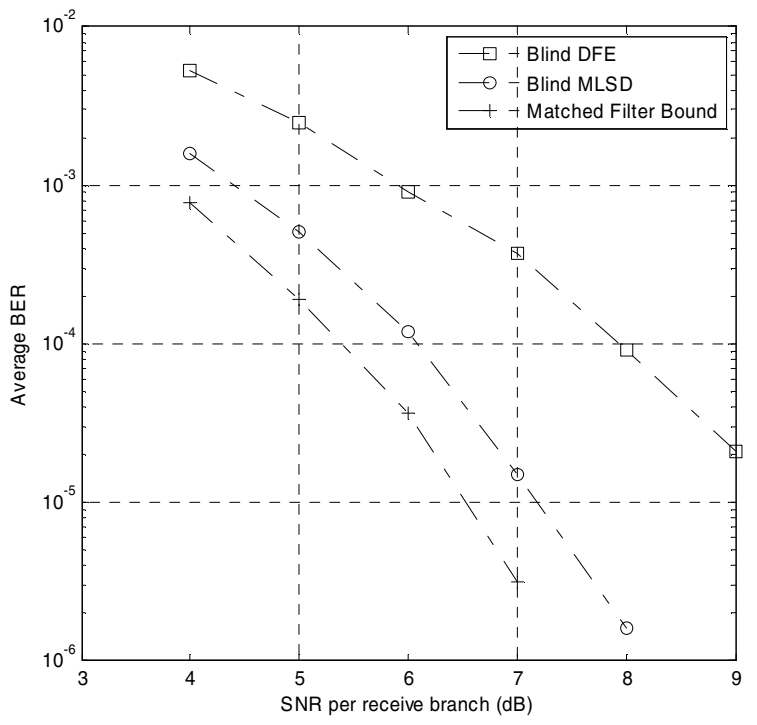

Fig. 4. BER comparison for the Novel Blind Scheme
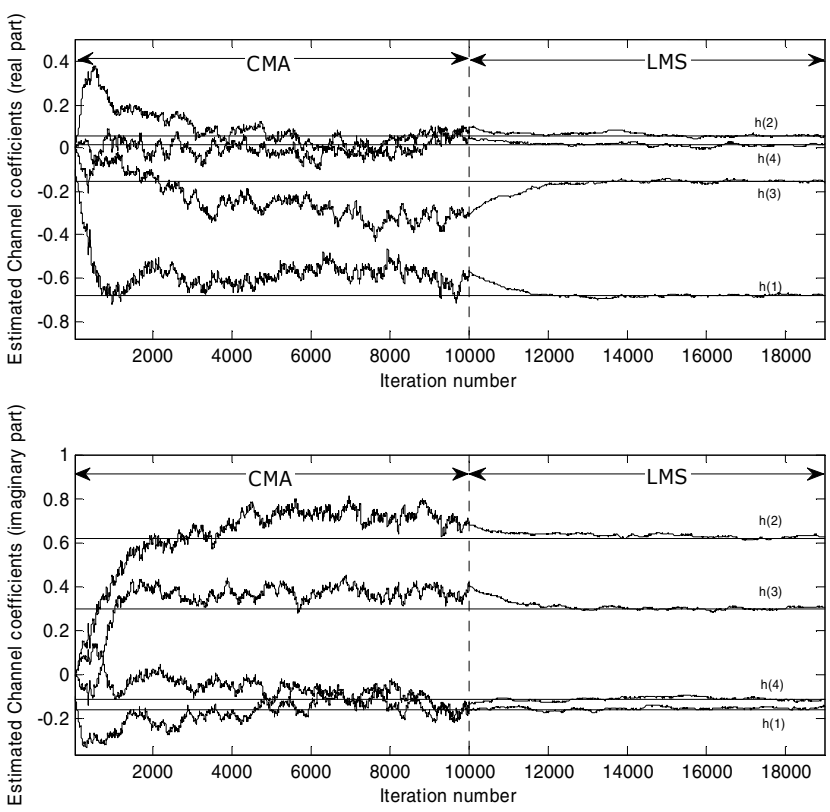

Fig. 5. Convergence of the CMA \& LMS algorithm to the true channel coefficients

DFE and further improvement is obtained by switching to the blind SIMO-MLSD setup. With the switching between the two novel blind techniques at $7 \mathrm{~dB}$, a more than 10 times better error rate is realized. Besides, the novel blind SIMO-MLSD setup has reached the same diversity order (which corresponds to the slope of the BER curve) same with the matched filter bound. To plot the matched filter bound a single shot transmission is assumed [6].

In order to show the convergence performance, Fig. 5 was plotted for the channel depicted in the second column of Table I at $5 \mathrm{~dB}$ SNR. Due to the normalization process in the algorithm the channel coefficients are scaled satisfying the normalization $\sum_{\mathrm{k}=0}^{\mathrm{L}-1}\left|h_{\mathrm{i}}[k]\right|^{2}=1$.

A total of 8 weights are plotted on Fig. 5 (4 real and corresponding 4 imaginary) where the true channel coefficients are shown with horizontal lines. On the plot the CMA \& LMS weights are shown changing over the iteration number and finally converging to the true values. The switch from the CMA algorithm to the LMS, algorithm was made at iteration number $1 \times 10^{5}$, where it is indicated by a dashed vertical line in Fig. 5. As can be seen from Fig. 5, through the use of the CMA, the channel coefficients were found to be within a small error and with an extra stage of the LMS algorithm the exact channel coefficients were identified. The step size of the LMS was kept small $\left(\eta=5 \times 10^{-4}\right)$ for smooth convergence.

In Fig.5, the Mean Squared Error (MSE) before switching to the LMS mode was $-10.689 \mathrm{~dB}$, and after the switching to the LMS algorithm it has further decreased down to $-30.579 \mathrm{~dB}$. For these calculations the MSE is defined as;

$$
M S E=\left[\sum_{\mathrm{i}=1}^{\mathrm{M}_{\mathrm{r}}}\left\|\hat{\mathbf{h}}_{\mathrm{i}}-\mathbf{h}_{\mathrm{i}}\right\|_{2}^{2}\right]\left[\sum_{\mathrm{i}=1}^{\mathrm{M}_{\mathrm{r}}}\left\|\mathbf{h}_{\mathrm{i}}\right\|_{2}^{2}\right]^{-1}
$$


where $\hat{\mathbf{h}}_{\mathrm{i}}$ is the channel vector estimated by the novel method and $\mathbf{h}_{i}$ is the vector of true channel coefficients. $\|\cdot\|_{2}$ represents the Euclidean norm operation.

\section{IV.CONCLUDING REMARKS}

This paper proposed a novel way for the blind estimation and blind equalization of SIMO channels. A blind multi-dimensional matched filter has been built by making use of the CMA, which is incorporated into a blind SIMO-MLSD setup. Therefore the optimum BER performance is achieved blindly, using the novel scheme. Moreover the estimates are further fine tuned by the LMS algorithm. Computer simulation results show that the novel blind structure is capable of identifying the matched filter of an unknown SIMO channel as well as blindly equalizing it. The results have also shown that the CMA alone is sufficient to be used for the equalization of SIMO channels, with enhanced error performance by introducing the LMS in the latter stages of the process.

\section{REFERENCES}

[1] G. Xu, H. Liu, L. Tong and T. Kailath, "A least squares approach to blind channel identification," IEEE Trans. Signal Processing, vol 43, pp. 2982-2993, Dec. 1995.
[2] E. Moulines, P. Duhamel, J.-F. Cardoso, and S. Mayrargue, "Subspace methods for the blind identification of multichannel FIR filters," IEEE Trans. Signal Processing, vol. 45. pp. 516-525, Feb. 1995.

[3] J.K. Tugnait, "Blind equalization and estimation of fir communications channels using fractional sampling", IEEE Trans. Commun., vol. 44, pp. 324-336, Mar. 1996.

[4] I. Ozcelik, I. Kale, B. Baykal, "A novel procedure for channel estimation through the CMA and its use in different MLSD setups", in IEEE ICECS'03, vol. 1, pp. 320-323, Dec 2003.

[5] D. Godard, "Self-recovering equalization and carrier tracking in two-dimensional data communications systems," IEEE Trans. Commun., vol. COM-28, no. 10, pp. 1867-1875, 1980.

[6] A. Paulraj, R. Nabar and D. Gore, Introduction to Space-Time Wireless Communications, Cambridge University Press, 2005.

[7] J. K. Hwang, C. H. Chen and M. C. Tsai, "Design and analytical error performance of MMSE-DFE receiver with spatial diversity", in IEEE SPAWC'01, pp. 13 -16, Mar. 2001.

[8] G. Li, Z. Ding, "New blind/semi-blind equalization algorithms for GSM systems," in IEEE SPAWC '99, pp. 66-69, May 1999.

[9] J. Proakis, Digital Communications, McGraw w-Hill Science, 2000.

[10] A. Scaglione, G. B. Giannakis, S. Barbarossa, "Redundant filterbank precoders and equalizers. II. Blind channel estimation, synchronization, and direct equalization", IEEE Trans. on Signal Process., vol. 47, pp. 2007-2022, July 1999. 